\title{
Tricuspid annular abnormalities following different surgical strategies in adults with corrected tetralogy of Fallot (Results from the CSONGRAD Registry and MAGYAR-Path Study)
}

\author{
Attila Nemes, Gergely Rácz, Árpád Kormányos, Nóra Ambrus, Kálmán Havasi \\ Department of Medicine, Medical Faculty, Albert Szent-Györgyi Clinical Center, University of Szeged, Szeged, Hungary \\ Contributions: (I) Conception and design: A Nemes; (II) Administrative support: A Nemes; (III) Provision of study materials or patients: A Nemes; \\ (IV) Collection and assembly of data: G Rácz, Á Kormányos; (V) Data analysis and interpretation: G Rácz, Á Kormányos, A Nemes; (VI) Manuscript \\ writing: All authors; (VII) Final approval of manuscript: All authors. \\ Correspondence to: Attila Nemes, MD, PhD, DSc, FESC. Department of Medicine, Medical Faculty, Albert Szent-Györgyi Clinical Center, University \\ of Szeged, Semmelweis street 8, P.O. Box 427, Szeged H-6725, Hungary. Email: nemes.attila@med.u-szeged.hu.
}

Background: Although there is an increasing number of patients with corrected tetralogy of Fallot (cTOF), who reach adulthood due to recent successful surgical procedures, some of them suffers from late complications, including functional tricuspid regurgitation (FTR). The purpose of the present study was to examine tricuspid annular (TA) abnormalities in adult cTOF patients and their relationship right atrial (RA) volumes respecting the cardiac cycle assessed by three-dimensional speckle-tracking echocardiography (3DSTE). Moreover, the effect of different surgical procedures on TA morphologic and functional parameters was examined as well.

Methods: The study comprised 24 cTOF patients $(32.8 \pm 13.5$ years, 9 males $)$ in which early palliative surgery and late total correction were performed in 12 cases (pcTOF), while early total reconstruction was performed in 12 subjects (etrTOF). Their results were compared to those of 33 matched healthy adults (36.7 \pm 7.2 years, 15 males).

Results: Dilated end-systolic and end-diastolic TA morphologic parameters and their body surface areaindexed counterpart could be detected in cTOF patients as compared to that of controls. TA fractional area change and TA fractional shortening proved to be reduced in cTOF patients as well as in etrTOF and pcTOF patients compared to controls. None of the TA morphologic and functional parameters showed any differences between etrTOF and pcTOF patients. Increased maximum, preatrial contraction and minimum RA volumes could be detected in cTOF patients compared to controls, which correlated with TA dimensions.

Conclusions: TA is dilated with reduced function in adult patients with cTOF. TA dilation is related to RA volumes. etrTOF and pcTOF patients have similar TA dimensions and TA functional properties.

Keywords: Fallot; tricuspid annulus; three-dimensional (3D); speckle-tracking; echocardiography

Submitted Apr 22, 2021. Accepted for publication Jul 16, 2021.

doi: $10.21037 / \mathrm{cdt}-21-256$

View this article at: https://dx.doi.org/10.21037/cdt-21-256 


\section{Introduction}

Tetralogy of Fallot (TOF) is a conotruncal malformation characterized by the presence of a multilevel right ventricular (RV) outflow tract obstruction, leading to RV hypertrophy, and a malalignment ventricular septal defect (VSD) with aortic override $(1,2)$. TOF is the most common congenital heart defect (CHD) accounting for 10\% of the cases and in unrepaired patients, cyanosis could develop due to right-toleft shunt. Two surgical strategies are used in TOF patients in general: early palliation by creating artificial left-to-right shunts to provide adequate pulmonary blood flow followed by a late total reconstruction (pcTOF), or an early total reconstruction is performed (etrTOF) (3). Although there is an increasing number of patients with corrected TOF (cTOF), who reach adulthood due to these successful surgical procedures, some of them suffer from late complications like functional tricuspid regurgitation (FTR) (4).

Three-dimensional speckle-tracking echocardiography (3DSTE) can be used not only for detailed volumetric and functional assessment of certain cardiac chambers at the same time using the same virtually acquired $3 \mathrm{D}$ echocardiographic datasets, but to determine annular dimensions and functional properties as well (5-7). The purpose of the present study was to examine tricuspid annular (TA) abnormalities in adult cTOF patients and their relationship with right atrial (RA) volumes respecting the cardiac cycle assessed by 3DSTE. Moreover, the effect of different surgical procedures on TA morphologic and functional parameters was also examined. We present the following article in accordance with the STROBE reporting checklist (available at https://dx.doi. org/10.21037/cdt-21-256).

\section{Methods}

\section{Patient population}

The present study comprised 24 adult patients with cTOF [mean age $32.8 \pm 13.5$ years, 15 males $(62 \%)$ ]. Data of these patients origin from a special registry for CHD patients treated and/or operated on at the Department of Pediatrics, Department of Heart Surgery, and 2nd Department of Medicine and Cardiology Center at the University of Szeged, Hungary since 1961. This registry is called as Registry of C(S)ONGenital cardiac Disease patients at the University of Szeged (CSONGRAD Registry) including data of more than 3,000 CHD patients (8). In 12 cTOF patients, early palliative surgery and late total correction were performed (pcTOF), while early total reconstruction was performed in $12 \mathrm{cTOF}$ patients (etrTOF). Their results were compared with those of 33 age- and gender-matched healthy volunteers, who had no known disorders, diseases or other pathological conditions, risk factors, did not receive any drugs and showed negative echocardiographic and electrocardiographic findings. All cTOF patients and healthy controls underwent transthoracic 2-dimensional (2D) Doppler echocardiography and 3DSTE. The present study serves as a part of the Motion Analysis of the heart and Great vessels by 3DSTE in Pathological cases (MAGYAR-Path) Study. Myocardial mechanics and valvular abnormalities were examined extensively in cTOF patients in this study among others. Informed consent was given by all cTOF patients and healthy controls. Institutional human research committee of the University of Szeged approved the study under the registration number 71/2011 (and updated versions). The study was conducted in accordance with the Declaration of Helsinki (as revised in 2013).

\section{M-mode and 2D Doppler echocardiography}

Toshiba Artida ${ }^{\text {TM }}$ cardiac ultrasound tool (Toshiba Medical Systems, Tokyo, Japan) attached with a 1-5 $\mathrm{MHz}$ PST-30SBP phased-array transducer was used for 2D Doppler echocardiographic examinations. Left ventricular (LV) dimensions and volumes, ejection fraction and left atrial diameter were measured according to the guidelines (9). Doppler echocardiography was used for quantification of valvular regurgitations and stenoses and for measurement of transmitral $\mathrm{E}$ and $\mathrm{A}$ waves as features of LV diastolic function. Tricuspid annular plane systolic excursion (TAPSE) was also determined by M-mode echocardiography (MME) as a quantitative feature of longitudinal movement of the TA and RV function $(10,11)$.

\section{DSTE}

3DSTE analysis was carried out using the same Toshiba Artida $^{\mathrm{TM}}$ cardiac ultrasound equipment (Toshiba Medical Systems, Tokyo, Japan) attached to a $1-4 \mathrm{MHz}$ matrix phased-array PST-25SX transducer (6). Two-step analysis was performed, first data acquisition was made of six wedgeshaped subvolumes within a single breath-hold from the transthoracic apical window, then the software created a full volume pyramid-shaped 3D dataset. The second step proved to be the data analysis, when using 3D Wall Motion Tracking software version 2.7 (Toshiba Medical Systems, Tokyo, Japan), data were presented in apical two- (AP2CH) 
and four-chamber (AP4CH) views.

\section{DSTE-derived analysis of the tricuspid annulus}

For analysis of TA parameters, planes were optimized on long-axis views to create an 'en-face' view of the TA on C7 short-axis view (12) (Figure 1). End-diastolic (just before tricuspid valve closure) and end-systolic (just before tricuspid valve opening) measurements were performed:

TA morphological parameters

(I) TA diameter $(\mathrm{TAD})=$ perpendicular line drawn from the peak of TA curvature to the middle of the straight TA border;

(II) TA area (TAA), measured by planimetry;

(III) TA perimeter (TAP), measured by planimetry;

TA functional features

(I) TA fractional shortening (TAFS) $=[$ (end-diastolic TAD - end-systolic TAD)/end-diastolic TAD] $\times 100$;

(II) TA fractional area change $($ TAFAC $)=[$ (enddiastolic TAA - end-systolic TAA)/end-diastolic $\mathrm{TAA}] \times 100$.

\section{DSTE-derived measurement of $R A$ volumes}

For measurement of RA volumetric parameters, a 3D model of the RA was created by using the same software demonstrated in recent studies (13). Shortly, following image optimizations, edges of the TA and the endocardial side of the superior RA region were defined and then the endocardial RA surface was reconstructed. Using the 3D cast of the RA, the following volumetric RA parameters were calculated:

(I) $\mathrm{V}_{\max }$ : end-systolic RA volume [largest left atrium (LA) volume before tricuspid valve opening];

(II) $\mathrm{V}_{\text {preA }}$ : early diastolic RA volume (detected on ECG before atrial contraction at time of $\mathrm{P}$ wave);

(III) $\mathrm{V}_{\text {min }}$ : late diastolic RA volume (smallest RA volume before tricuspid valve closure).

\section{Statistical analysis}

Continuous and categorical variables are presented as mean values \pm standard deviation and frequencies and percentages, respectively. Levene's test was used to assess homogeneity of variances. Student's $t$-test was used in normally distributed data, while Mann-Whitney Wilcoxon test was used in case of not normally distributed data. Chi-squared test and Fisher's exact test were used for statistical analysis of categorical variables. All tests proved to be 2 -sided and $\mathrm{P}<0.05$ was considered to be statistically significant. Medcalc software package was used for statistical analysis (MedCalc, Mariakerke, Belgium).

\section{Results}

\section{Clinical data}

From the 24 adult cTOF patients, 12 had palliative surgery first [modified Blalock-Taussig ( $n=9)$, Waterston-Cooley $(n=1)$ shunts, or Brock procedure $(n=2)]$ (mean age at the palliation: $2.4 \pm 2.1$ years, median age: 1.4 years, interquartile range: 3.5 years) followed by a late total correction (mean age at the total correction: $6.9 \pm 6.7$ years, median age: 5.5 years, interquartile range: 6.5 years) (pcTOF group). Early total correction was performed in another 12 TOF patients (mean age at the repair: $3.9 \pm 3.1$ years, median age: 5.0 years, interquartile range: 5.0 years) (etrTOF group). Demographic data and risk factors and their distribution in the cTOF group and subgroups and in controls are presented in Table 1.

\section{D echocardiographic data}

Left atrial and LV dimensions in controls and in patients with cTOF and in subgroups are presented in Table 1. TAPSE proved to be $18.7 \pm 3.2 \mathrm{~mm}$ in cTOF patients, which did not differ between etrTOF and pcTOF patients $(18.8 \pm 2.0$ vs. $18.6 \pm 4.1 \mathrm{~mm}, \mathrm{P}=\mathrm{ns})$. Valvular regurgitations and their severity expressed in grades are presented in Table 1. Mean RV pressure was $14.3 \pm 2.1 \mathrm{mmHg}$ without residual pulmonary stenosis or narrowing of a pulmonary artery branch.

\section{DSTE}

Dilated end-systolic and end-diastolic TA morphologic parameters and their BSA-indexed counterpart could be detected in cTOF patients as compared to controls (Table 2). TAFAC and TAFS proved to be reduced in cTOF patients as well as in etrTOF and pcTOF patients compared to controls. None of TA morphologic and functional parameter showed any differences between etrTOF and pcTOF patients. Increased maximum $(64.0 \pm 32.1$ vs. $36.9 \pm 9.5 \mathrm{~mL}$, $\mathrm{P}<0.05)$, preatrial contraction $(56.2 \pm 30.9$ vs. $30.5 \pm 9.3 \mathrm{~mL}$, $\mathrm{P}<0.05)$ and minimum $(48.6 \pm 31.9$ vs. $24.0 \pm 8.4 \mathrm{~mL}$, $\mathrm{P}<0.05) \mathrm{RA}$ volumes could be detected in cTOF patients 
Table 1 Baseline demographic and 2D Doppler echocardiographic data in patients with tetralogy of Fallot and controls

\begin{tabular}{|c|c|c|c|c|}
\hline Parameters & Controls $(n=33)$ & All cTOF patients $(n=24)$ & EtrTOF patients $(n=12)$ & PcTOF patients $(n=12)$ \\
\hline Age (years) & $36.7 \pm 7.2$ & $32.8 \pm 13.5$ & $32.6 \pm 14.5$ & $33.7 \pm 15.0$ \\
\hline Male gender [\%] & $15[45]$ & $9[41]$ & $7[58]$ & $3[25]$ \\
\hline Hypertension [\%] & $0[0]$ & $5[22]^{*}$ & $2[17]$ & $4[33]^{*}$ \\
\hline Hypercholesterolaemia [\%] & $0[0]$ & $1[4]$ & $0[0]$ & $1[8]$ \\
\hline \multicolumn{5}{|l|}{ Two-dimensional echocardiography } \\
\hline LA diameter (mm) & $38.4 \pm 3.2$ & $41.3 \pm 6.5$ & $41.2 \pm 7.2$ & $41.3 \pm 6.0$ \\
\hline LV end-diastolic diameter (mm) & $48.5 \pm 2.8$ & $50.1 \pm 14.1$ & $47.3 \pm 5.6$ & $47.9 \pm 5.6$ \\
\hline LV end-systolic volume (mL) & $37.9 \pm 7.0$ & $38.0 \pm 19.9$ & $37.7 \pm 20.0$ & $38.4 \pm 20.6$ \\
\hline Interventricular septum (mm) & $9.2 \pm 1.0$ & $9.8 \pm 1.5$ & $9.5 \pm 1.1$ & $10.1 \pm 1.9$ \\
\hline LV posterior wall (mm) & $8.9 \pm 1.0$ & $9.5 \pm 1.4$ & $9.3 \pm 1.4$ & $9.8 \pm 1.4$ \\
\hline LV ejection fraction (\%) & $65.1 \pm 2.4$ & $65.5 \pm 8.7$ & $65.5 \pm 6.3$ & $65.5 \pm 10.9$ \\
\hline \multicolumn{5}{|l|}{ Mitral regurgitation [\%] } \\
\hline Grade 1 & $0[0]$ & $13[54]^{\star}$ & $5[41]^{\star}$ & $8[75]^{*}$ \\
\hline Grade 2 & $0[0]$ & $2[8]$ & $1[8]$ & $1[8]$ \\
\hline \multicolumn{5}{|l|}{ Tricuspid regurgitation [\%] } \\
\hline
\end{tabular}

*, P<0.05 vs. controls. 2D, two-dimensional; cTOF, corrected tetralogy of Fallot; etrTOF, TOF-early total reconstruction; pcTOF, TOF-early palliation, late correction; LA, left atrial; LV, left ventricular.

compared to controls.

\section{Correlations}

End-diastolic TAD and TAA correlated with $V \max (r=0.58$, $\mathrm{P}=0.05$ and $\mathrm{r}=0.63, \mathrm{P}=0.04$, respectively), VpreA $(\mathrm{r}=0.50$, $\mathrm{P}=0.05$ and $\mathrm{r}=0.57, \mathrm{P}=0.04$, respectively) and $\mathrm{Vmin}(\mathrm{r}=0.76$, $\mathrm{P}=0.03$ and $\mathrm{r}=0.76, \mathrm{P}=0.05$, respectively). Similarly, endsystolic TAD and TAA correlated with $\mathrm{Vmax}(\mathrm{r}=0.57, \mathrm{P}=0.05$ and $r=0.63, P=0.05$, respectively), $\operatorname{VpreA}(r=0.59, P=0.03$ and $\mathrm{r}=0.55, \mathrm{P}=0.05$, respectively) and $\mathrm{V} \min (\mathrm{r}=0.76, \mathrm{P}=0.04$ and $\mathrm{r}=0.73, \mathrm{P}=0.05$, respectively), as well. End-diastolic and endsystolic TAP showed correlations with $\mathrm{V}_{\min }$ only $(\mathrm{r}=0.56$,
$\mathrm{P}=0.05$ and $\mathrm{r}=0.66, \mathrm{P}=0.04$, respectively). Only TAFS correlated with $\mathrm{V}_{\min }(\mathrm{r}=-0.45, \mathrm{P}=0.05)$, other correlations between TA functional properties and RA volumes could not be detected. No correlations could be demonstrated between TAPSE and 3DSTE-derived TA dimensions and functional properties (TAFAC, TAFS) in cTOF patients.

\section{Discussion}

To the best of the authors' knowledge, this is the first time to examine 3DSTE-derived TA abnormalities in adult cTOF patients and their relationship with RA volumes. Several disorders like cTOF itself may lead to FTR through 
Table 2 Comparison of 3D speckle-tracking echocardiography-derived TA morphological and functional parameters between adult patients with TOF and controls

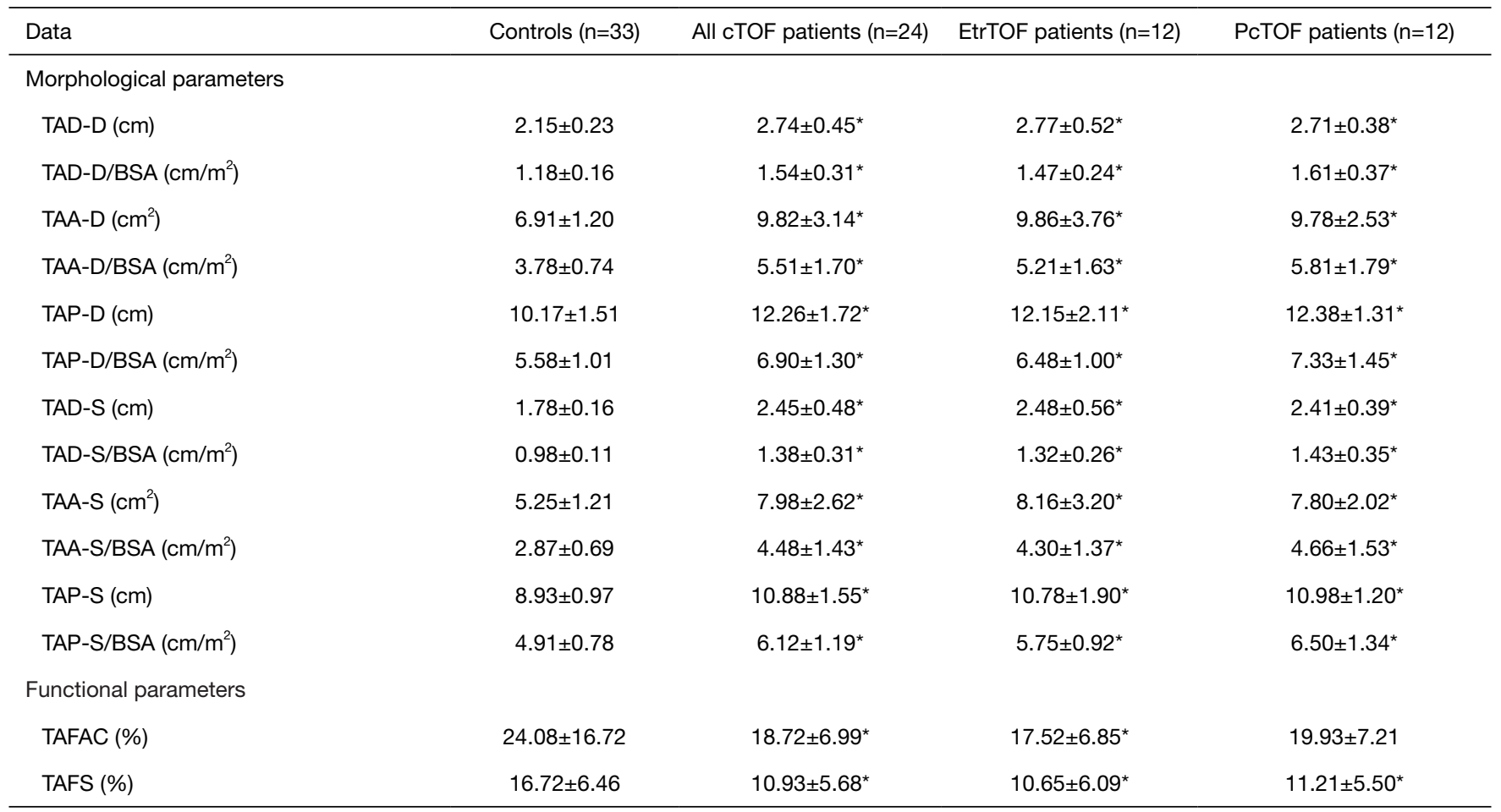

${ }^{*}, \mathrm{P}<0.05$ vs. controls. 3D, three-dimensional; BSA, body surface area; TA, tricuspid annular; TOF, tetralogy of Fallot; cTOF, corrected tetralogy of Fallot; etrTOF, TOF-early total reconstruction; pcTOF, TOF-early palliation, late correction; TAD-D, end-diastolic tricuspid annular diameter; TAA-D, end-diastolic tricuspid annular area; TAP-D, end-diastolic tricuspid annular perimeter; TAD-S, end-systolic tricuspid annular diameter; TAA-S, end-systolic tricuspid annular area; TAP-S, end-systolic tricuspid annular perimeter; TAFAC, tricuspid annular fractional area change; TAFS, tricuspid annular fractional shortening.

TA dilation. In patients with atrial fibrillation, RA dilation were more important than RV dilation to determine TA dilation and subsequent FTR development. Minimum RA volume and TA area were directly correlated to FTR severity (14). Moreover, RA volume was found to be a major determinant of TA area, and RA enlargement is an important mechanism of TA dilation in FTR irrespective of the cardiac rhythm and RV loading conditions (15). In a recent 3DSTE study, detailed analysis showed increased RA volumes with reduced RA emptying fractions together with unchanged RA stroke volumes respecting the cardiac cycle in cTOF patients (5). In the present study, FTR was significantly common in cTOF patients with more pronounced presence in pcTOF cases. Both enddiastolic and end-systolic TA dimensions were found to be dilated in cTOF as compared to those of controls, and were accompanied with TA functional impairment. No differences could be demonstrated, however, in TA morphological and functional properties according to the surgical procedure performed. Moreover, strong correlations could be demonstrated between RA volumes and TA dimensions in cTOF suggesting a determinative relationship between these parameters.

Old-fashioned MME and the most recent 3DSTE together allows complex spatial analysis of the TA movement thought the cardiac cycle. Vertical movement of the TA represented by TAPSE and decreased horizontal 'sphincter-like' TA motion represented by TAFAC and TAFS did not correlate with each other in cTOF in this study. These results could suggest complexity of deterioration of TA functions in cTOF, which requires further examinations on the right heart function.

According to the surgical procedures used for TOF corrections, beneficial results could be demonstrated in recent studies related to $\mathrm{LV}$ contractility represented by LV strains (16) and LV rotational mechanics (17) and MA morphology and function (18) in etrTOF patients compared to pcTOF patients. Similar findings could not be detected 


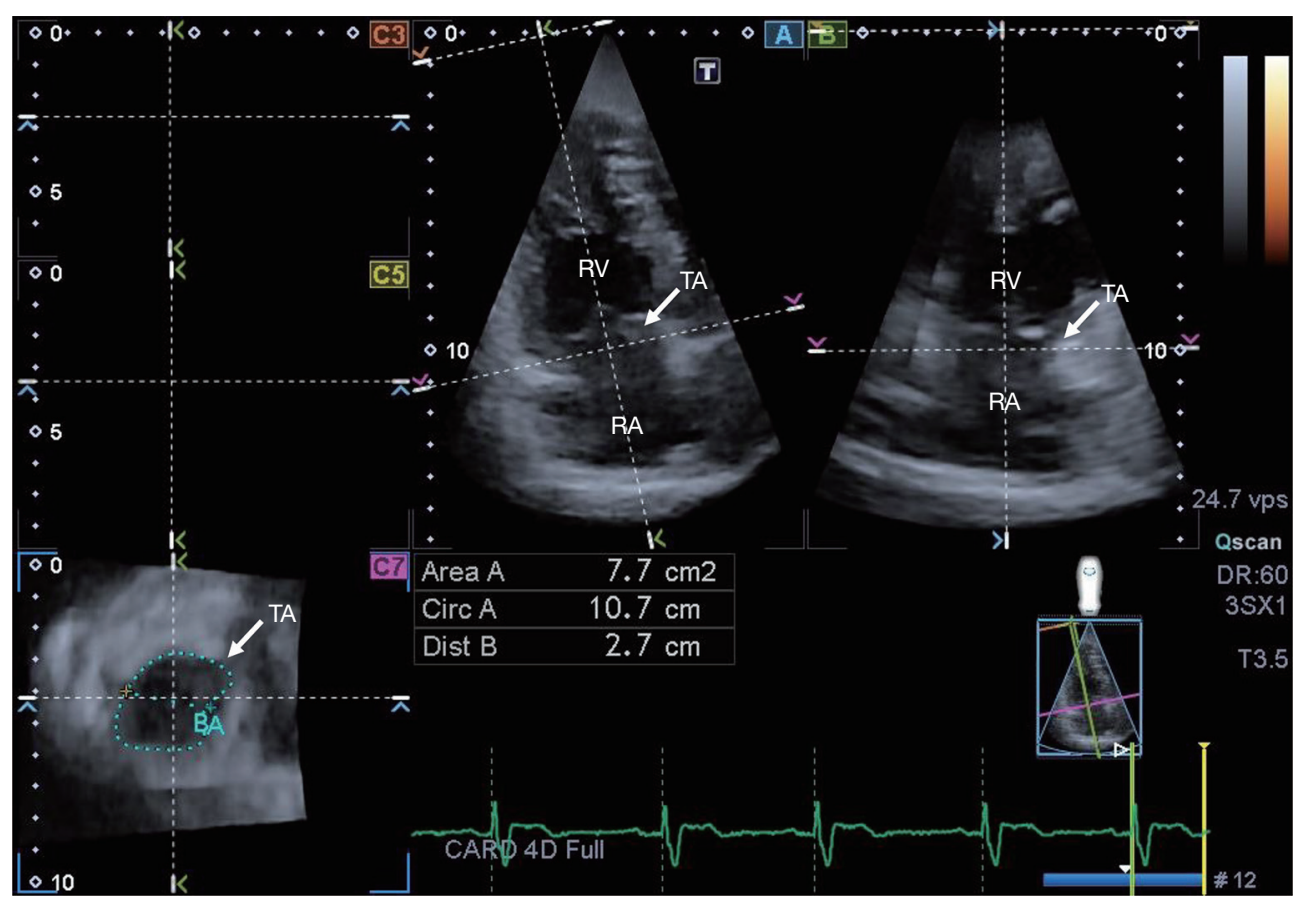

Figure 1 Images from a 3D echocardiographic full-volume dataset in a patient with corrected tetralogy of Fallot: (A) apical four-chamber view; (B) apical two-chamber view and a cross sectional view at the level of the tricuspid annulus; (C7) optimized on apical four- and twochamber views. The arrow represents the TA plane on the long- $(\mathrm{A}, \mathrm{B})$ and short-axis $(\mathrm{C} 7)$ images. 3D, three-dimensional; RA, right atrium; $\mathrm{RV}$, right ventricle; TA, tricuspid annular.

in TA parameters between the subgroups examined. These results could suggest fixed clinical situation on the right heart, but further studies are warranted to confirm our findings.

\section{Limitation section}

The following important limitations were present:

(I) $2 \mathrm{D}$ echocardiography has higher temporal and spatial resolution than 3 DSTE, which could affect the results;

(II) Interchamber and atrio-ventricular interactions could affect TA function, more detailed analysis would have strengthened the results;

(III) Although TOF has a prevalence of 3-6 per 10,000 births, only limited number $(n=24)$ of adult patients with cTOF were involved into the present study $(1,2)$;

(IV) The normal reference values of 3DSTE-derived TA parameters are not available in the literature at this moment.

\section{Conclusions}

TA is dilated in cTOF with reduced function in adult patients with cTOF. TA dilation is related to RA volumes. etrTOF and pcTOF patients have similar TA dimensions and TA functional properties.

\section{Acknowledgments}

Funding: None.

\section{Footnote}

Provenance and Peer Review: This article was commissioned by the Guest Editors (Yskert von Kodolitsch, Harald Kaemmerer, Koichiro Niwa) for the series "Current Management Aspects in Adult Congenital Heart Disease (ACHD): Part IV" published in Cardiovascular Diagnosis and Therapy. The article has undergone external peer review.

Reporting Checklist: The authors have completed the 
STROBE reporting checklist. Available at https://dx.doi. org/10.21037/cdt-21-256

Data Sharing Statement: Available at https://dx.doi. org/10.21037/cdt-21-256

Conflict of Interest: All authors have completed the ICMJE uniform disclosure form (available at https:// dx.doi.org/10.21037/cdt-21-256). The series "Current Management Aspects in Adult Congenital Heart Disease (ACHD): Part IV" was commissioned by the editorial office without any funding or sponsorship. The authors have no other conflicts of interest to declare.

Ethical Statement: The authors are accountable for all aspects of the work in ensuring that questions related to the accuracy or integrity of any part of the work are appropriately investigated and resolved. Informed consent was given by all cTOF patients and healthy controls. Institutional human research committee of the University of Szeged approved the study under the registration number $71 / 2011$ (and updated versions). The study was conducted in accordance with the Declaration of Helsinki (as revised in 2013).

Open Access Statement: This is an Open Access article distributed in accordance with the Creative Commons Attribution-NonCommercial-NoDerivs 4.0 International License (CC BY-NC-ND 4.0), which permits the noncommercial replication and distribution of the article with the strict proviso that no changes or edits are made and the original work is properly cited (including links to both the formal publication through the relevant DOI and the license). See: https://creativecommons.org/licenses/by-nc-nd/4.0/.

\section{References}

1. Bedair R, Iriart X. Educational series in congenital heart disease: Tetralogy of Fallot: diagnosis to long-term followup. Echo Res Pract 2019;6:R9-R23.

2. Apitz C, Webb GD, Redington AN. Tetralogy of Fallot. Lancet 2009;374:1462-71.

3. Al Habib HF, Jacobs JP, Mavroudis C, et al. Contemporary patterns of management of tetralogy of Fallot: data from the Society of Thoracic Surgeons Database. Ann Thorac Surg 2010;90:813-9; discussion 819-20.

4. Yucel E, Bertrand PB, Churchill JL, et al. The tricuspid valve in review: anatomy, pathophysiology and echocardiographic assessment with focus on functional tricuspid regurgitation. J Thorac Dis 2020;12:2945-54.

5. Nemes A, Havasi K, Domsik P, et al. Evaluation of right atrial dysfunction in patients with corrected tetralogy of Fallot using 3D speckle-tracking echocardiography. Insights from the CSONGRAD Registry and MAGYARPath Study. Herz 2015;40:980-8.

6. Nemes A, Kalapos A, Domsik P, et al. Three-dimensional speckle-tracking echocardiography -- a further step in non-invasive three-dimensional cardiac imaging. Orv Hetil 2012;153:1570-7.

7. Nemes A, Kovács Z, Kormányos Á, et al. The mitral annulus in lipedema: Insights from the three-dimensional speckle-tracking echocardiographic MAGYAR-Path Study. Echocardiography 2019;36:1482-91.

8. Havasi K, Kalapos A, Berek K, et al. More than 50 years' experience in the treatment of patients with congenital heart disease at a Hungarian university hospital. Orv Hetil 2015;156:794-800.

9. Lang RM, Badano LP, Mor-Avi V, et al. Recommendations for cardiac chamber quantification by echocardiography in adults: an update from the American Society of Echocardiography and the European Association of Cardiovascular Imaging. Eur Heart J Cardiovasc Imaging 2015;16:233-70.

10. Aloia E, Cameli M, D'Ascenzi F, et al. TAPSE: An old but useful tool in different diseases. Int J Cardiol 2016;225:177-83.

11. Rudski LG, Lai WW, Afilalo J, et al. Guidelines for the echocardiographic assessment of the right heart in adults: a report from the American Society of Echocardiography endorsed by the European Association of Echocardiography, a registered branch of the European Society of Cardiology, and the Canadian Society of Echocardiography. J Am Soc Echocardiogr 2010;23:685713; quiz 786-8.

12. Anwar AM, Soliman OI, Nemes A, et al. Value of assessment of tricuspid annulus: real-time threedimensional echocardiography and magnetic resonance imaging. Int J Cardiovasc Imaging 2007;23:701-5.

13. Nemes A, Kormányos Á, Domsik P, et al. Normal reference values of three-dimensional speckle-tracking echocardiography-derived right atrial volumes and volume-based functional properties in healthy adults (Insights from the MAGYAR-Healthy Study). J Clin Ultrasound 2020;48:263-8.

14. Guta AC, Badano LP, Tomaselli M, et al. The 
Pathophysiological Link between Right Atrial

Remodeling and Functional Tricuspid Regurgitation in Patients with Atrial Fibrillation: A Three-Dimensional Echocardiography Study. J Am Soc Echocardiogr 2021;34:585-594.e1.

15. Muraru D, Addetia K, Guta AC, et al. Right atrial volume is a major determinant of tricuspid annulus area in functional tricuspid regurgitation: a three-dimensional echocardiographic study. Eur Heart J Cardiovasc Imaging 2021;22:660-9.

16. Rácz G, Kormányos Á, Domsik P, et al. Left ventricular strains correlate with aortic elastic properties in adult patients with corrected tetralogy of Fallot (Results from the CSONGRAD Registry and MAGYAR-Path Study).

Cite this article as: Nemes A, Rácz G, Kormányos Á, Ambrus N, Havasi K. Tricuspid annular abnormalities following different surgical strategies in adults with corrected tetralogy of Fallot (Results from the CSONGRAD Registry and MAGYAR-Path Study). Cardiovasc Diagn Ther 2021;11(6):1276-1283. doi: 10.21037/cdt-21-256
Cardiovasc Diagn Ther 2021;11:611-22.

17. Nemes A, Rácz G, Kormányos Á, et al. Left ventricular rotational abnormalities in adult patients with corrected tetralogy of Fallot following different surgical procedures (Results from the CSONGRAD Registry and MAGYARPath Study). Cardiovasc Diagn Ther 2021;11:623-30.

18. Nemes A, Kormányos Á, Havasi K, et al. Mitral annulus is enlarged and functionally impaired in adult patients with repaired tetralogy of Fallot as assessed by threedimensional speckle-tracking echocardiography-results from the CSONGRAD Registry and MAGYAR-Path Study. Cardiovasc Diagn Ther 2019;9:S221-7. 